\title{
3D-numerische Modellansätze für die Berechnung von Lawineneinstößen in Speicher
}

\author{
Roman Gabl · Jakob Seibl · Manfred Pfeifer · Bernhard Gems · Markus Aufleger
}

Online publiziert: 30. September 2016

(c) Der/die Autor(en) 2016. Dieser Artikel ist eine Open-Access-Publikation.

\begin{abstract}
Zusammenfassung Speicherbecken im Pumpbetrieb oder Beschneiungsspeicher können in Zeiträumen großer

\section{Concepts to simulate avalanche impacts into a reservoir based on 3D-numerics} Lawinengefahr einen hohen Wasserstand aufweisen. In Hinblick auf eine Gefahrenbetrachtung und Risikoabschätzung ist deshalb auch ein möglicher Einstoß einer Lawine in einen gefüllten Speicher zu untersuchen. Der Einstoß einer großen Lawine kann dabei eine Impulswelle erzeugen, welche eine Gefährdung für den Uferbereich, das Absperrbauwerk und in weiterer Folge auch für die Unterlieger darstellt. Um den Effekt abschätzen und mögliche Gegenmaßnahmen (Speicherabsenkung oder bauliche Maßnahmen) überprüfen zu können, haben sich Modellversuche bewährt. Vermehrt kommen aber auch numerische Methoden zum Einsatz, die von allgemein gültigen Berechnungstabellen bis hin zu projektspezifischen 3D-numerischen Berechnungen reichen können.

Der Beitrag fasst die Entwicklungen bei der Modellbildung für die Randbedingung und Erzeugung dieser Impulswelle für die 3D-numerische Software FLOW-3D zusammen. Diese umfassen die Definition von (i) Partikelgemischen, (ii) dynamischen Festkörpern und (iii) ausschließlich aus Wasser bestehenden Fluidkörpern in der Sturzbahn der Lawine. Mögliche Konzepte sowie deren Vor- und Nachteile werden aufgezeigt. Zudem wird die Möglichkeit der Modifikation der „Wasserlawine“ durch Modifikation der Startdichte vorgestellt.

Dr. R. Gabl $(\bowtie) \cdot$ DI J. Seibl ·

DI M. Pfeifer · Dr. B. Gems .

Univ.-Prof. Dr.-Ing. M. Aufleger

Universität Innsbruck,

Arbeitsbereich Wasserbau,

Technikerstraße 13, 6020 Innsbruck,

Österreich

roman.gabl@uibk.ac.at
Abstract Avalanche impacts into reservoirs can cause an impulse wave which endanger the area around the reservoir, the dam itself and as well the area located downstream of the reservoir. Especially reservoirs for artificial snow production or pump storage can be filled up to high water levels in periods with significant avalanche hazard impulse wave scenarios is part of the limitation of the maximum permitted water level in the reservoir under specific weather condition or structural measures. To quantify such an impulse wave, general equations, scale model tests or numerical simulations can be applied.

Within the present paper focus is put on different approaches to model an avalanche impact into a reservoir and the corresponding impulse wave generation by use of the 3D-numerical simulation tool FLOW-3D. Different modelling concepts are presented and discussed, consisting of (i) particle-water-mixtures, (ii) moving objects and (iii) pure water avalanches. In addition to this review on the development, preliminary results of the advanced water avalanche are compared to the equations provided by the ETH Zürich. Therefore, the possibility to define a different density for each initial fluid region is used and two parameters (initial velocity of the avalanche at the start and the adapted density of the model avalanche) are varied.

\section{Einleitung}

Die besondere Gefährdung von bestehenden oder auch zukünftigen Wohngebieten und Infrastruktureinrichtungen durch Naturgefahrenprozesse im alpinen Raum hat zur Folge, dass abhängig von den potenziellen Einwirpotential. The investigation of possible global risk analysis and can lead to a kungen ein entsprechendes Risikomanagement betrieben werden muss. Dieses umfasst neben der Modellierung und Analyse möglicher gravitativer Naturgefahren (zum Beispiel Hochwasser, murartige Prozesse, Steinschlag, Lawinen) weitere aktive und passive Maßnahmen, um das Gefahren- bzw. das Schadenspotenzial von Ereignissen möglichst zu minimieren. Angewandte Konzepte umfassen planerische und organisatorische Maßnahmen, beispielsweise die Gefahrenzonenplanung oder die Ausarbeitung von Evakuierungsplänen, und technische bzw. forstlichbiologische Maßnahmen wie die Errichtung von Schutzbauten, den Objektschutz oder die Schutzwaldbewirtschaftung (Bergmeister et al. 2009; Gems et al. 2016; Möderl et al. 2008; Sauermoser et al. 2016; Kienholz et al. 2004).

Die Herausforderungen bei der Bemessung eines extremen Ereignisses sind sehr vielfältig und beginnen bereits bei den potenziellen Unsicherheiten der Eingangsdaten, zumal sich diese auf Abschätzungen und Extrapolationen beziehen. Für die Abschätzung von Ereignisintensitäten mit großen Jährlichkeiten gibt es in der Regel kaum Erfahrungswerte oder Daten, um ein entsprechendes Modell zu kalibrieren. Zudem verursachen Naturgefahrenprozesse mit großen Jährlichkeiten häufig Folgeprozesse, die in ihrer Charakteristik und Intensität schwer $\mathrm{zu}$ prognostizieren sind. Beispielsweise ist bei einem Dammbruch zu erwarten, dass das in großen Mengen und mit sehr hohen Geschwindigkeiten ausströmende Wasser erhebliche Mengen an Festoffen entlang der Fließwege mobilisiert. Dies kann wiederum zum Beispiel zu Verklausungen bei Brücken führen und damit einen temporären Aufstau oder eine partielle Umleitung der Wassermassen zur Folge haben (Gabl et al. 2014b). Massive Umlagerungen können auch zu Hangrutschungen führen und weiteren Schaden anrichten. In der Regel werden komplexe (Folge-) 
Tab. 1 Eingangswertezur Bestimmung des Impulsproduktionsparameters $P$ (Heller et al. 2009)

\begin{tabular}{|l|l|}
\hline$v_{\mathrm{s}}$ & $\begin{array}{l}\text { Rutscheintauchge- } \\
\text { schwindigkeit }\left[\mathrm{m} \mathrm{s}^{-1}\right]\end{array}$ \\
\hline$g$ & Erdbeschleunigung $\left[\mathrm{m} \mathrm{s}^{-2}\right]$ \\
\hline$h$ & Ruhewassertiefe im Speicher [m] \\
\hline$s$ & Rutschmächtigkeit [m] \\
\hline$\rho_{\mathrm{s}}$ & Rutschdichte $\left[\mathrm{kg} \mathrm{m}^{-3}\right]$ \\
\hline$V_{\mathrm{s}}$ & Rutschvolumen $\left[\mathrm{m}^{-3}\right]$ \\
\hline$\rho w$ & Wasserdichte $\left[\mathrm{kg} \mathrm{m}^{-3}\right]$ \\
\hline$b$ & Rutschbreite $[\mathrm{m}]$ \\
\hline$\alpha$ & Hangwinkel $\left[{ }^{\circ}\right]$
\end{tabular}

Prozesse entweder vernachlässigt oder sehr vereinfacht betrachtet. Dabei ist aber die Gültigkeit der Vereinfachung immer zu überprüfen. Beispielhaft sei hier die einem Dammbruch zugrunde liegende Bestimmung des maximalen Abflusses unter der Annahme eines plötzlichen Bruchs erwähnt. Für eine solche Abschätzung kann die Formel nach Ritter (Ritter 1892) herangezogen werden, wobei sich diese Untersuchung auf Rinnenversuche stützt, bei der der gesamte Gerinnequerschnitt plötzlich freigegeben wird. Seibl et al. (2014) konnten zeigen, dass die Form des betroffenen Speichers einen deutlichen Einfluss auf den maximalen Abfluss hat und die Werte von Ritter überschritten werden können, sofern eine im räumlichen Bezug zur angenommen Öffnung ungünstige Speicherform vorherrscht. Eine Eignungsprüfung des Modellierungsansatzes für die jeweilige konkrete Untersuchung kombiniert mit einer breiten Sensitivitätsstudie ist somit in den meisten Fällen unausweichlich.

Um die Zuverlässigkeit der Abschätzung von extremen Ereignissen zu verbessern, können unterschiedlichste numerische Werkzeuge, aber auch Modelluntersuchungen im Labor genutzt werden. Dabei kommt vermehrt eine Kombination aus unterschiedlichsten Methoden zum Einsatz, bei der sich im Idealfall die Vorteile jeder Methode ergänzen (Faber et al. 2012; De Cesare et al. 2012; Gems et al. 2014; Gabl et al. 2013, 2014b). Prinzipiell ist aber bei Untersuchungen extremer Ereignisse ein möglichst einfacher Ansatz zu bevorzugen, welcher auch in Relation zur vorhandenen Datenlage steht. Damit werden zum einen keine überhöhten Genauigkeitsansprüche provoziert und zum anderen wird eine möglichst breite und umfangreiche (Eingangs-)Parameterstudie ermöglicht.
Eine Form gravitativer Naturgefahren und Gegenstand des vorliegenden Beitrages sind Lawinen. Der Beitrag fokussiert sich dabei nicht direkt auf die Modellierung von Lawinenprozessen, sondern setzt die Modellierung der Lawine an sich voraus und untersucht einen potenziellen Einstoß in ein Speicherbecken als indirekte Einwirkung eines Lawinenabgangs. Als Folge der unmittelbaren Wasserverdrängung beim Auftreffen einer Lawine auf die ruhende Wasseroberfläche entsteht eine Impulswelle, die sich im Speicher gleichermaßen in alle Richtungen ausbreitet. Entsprechend dem Ausmaß und der Fortpflanzung inklusive möglicher Reflexion einer solchen Welle kann ein solches Ereignis eine Gefährdung für das Absperrbauwerk der zugehörigen Talsperre oder auch für die umliegende Infrastruktur und Siedlungen darstellen. Im Fokus stehen dabei insbesondere kleinere Speicher, die für Beschneiungsanlagen (Czerny und Eistert 2014) genutzt werden, aber auch große Speicher von Pumpspeicheranlagen, die im Gegensatz zu den konventionell bewirtschafteten Jahresspeichern auch im Zeitraum großer Lawinengefahr eine hohe Staulage aufweisen können. Eine Möglichkeit, das Gefahrenpotenzial zu reduzieren, besteht in der (zeitlichen) Limitierung des maximal zulässigen Stauspiegels im Speicher und somit in der Verhinderung einer möglichen Überströmung des Absperrbauwerks.

An der ETH Zürich wurden und werden zur angesprochenen Thematik sehr breite Grundlagenuntersuchungen im Rahmen mehrerer Dissertationen und Forschungsprojekten durchgeführt, die unter anderem im Bemessungsbehelf von Heller et al. (2009) zusammengefasst wurden. Neben der Generierung und Ausbreitung von masseninduzierten Impulswellen in Speichern wird auch das Überströmen und Auflaufen auf Gegenhängen untersucht (Fuchs und Hager 2012; Fuchs et al. 2010), das auch zu einem Abtrag des Dammes führen kann (Müller et al. 2016). Diese Untersuchungen bieten belastbare Bemessungsansätze für gerade und kreisförmige Ausbreitungen von Impulswellen sowie deren Kombinationen. Um zusätzlich den Einfluss und die Wirkung von komplexeren Speicherformen, separierten Lawinen, Wellenbrechern oder auch zum Beispiel Hochwasserentlastungsanlagen berücksichtigen $\mathrm{zu}$ können, sind ge- gebenenfalls weitere projektspezifische Untersuchungen notwendig. Solche Fragestellungen werden in der Regel basierend auf einem skalierten Labormodell beantwortet. Beispielhaft kann die an der ETH Zürich durchgeführte Untersuchung des Speichers Kühtai genannt werden (Fuchs et al. 2011). Neben diesem erprobten Ansatz bietet auch die 3D-Numerik die Möglichkeit, solche Fragestellungen zu untersuchen. Der vorliegende Beitrag fasst die Entwicklungen und Erfahrungen zusammen, die in diesem Kontext mit der Anwendung der kommerziellen 3D-numerischen Software FLOW-3D an der Universität Innsbruck gemacht wurden, und präsentiert dabei auch neue Erkenntnisse aus der Arbeit von Pfeifer (2016).

\section{Möglichkeiten der Modellierung von Lawineneinstößen in Speicher}

\subsection{Modellversuche und Grundlagen}

Im Zuge einer Laboruntersuchung eines Lawineneinstoßes in einen Speicher wird die Topografie skaliert im Labor nachgebaut und der Speicher entsprechend gefüllt. Dabei ist darauf zu achten, dass die Wassertiefe möglichst größer als $0,2 \mathrm{~m}$ ist, wodurch mögliche unerwünschte Maßstabseffekte vermieden werden können (Heller et al. 2008). Die Lawine wird für solche Untersuchungen vereinfacht dargestellt, wobei in der Regel entweder Granulate oder kompakte Festkörper (Heller und Spinneken 2015; Heller et al. 2016) zur Anwendung kommen. Die Beschleunigung der Modelllawine erfolgt meist in einer steilen Rinne, deren Ende in der Regel am Einstoßpunkt der Lawine angeordnet (Gabl et al. 2010, 2014b) oder leicht erhöht ist, wodurch eine Anpassung der Modelllawine an das gegebene Gelände ermöglicht wird (Fuchs et al. 2011).

Der Einfluss der Granulatdichte und -porosität wurde unter anderem von Zweifel (2004) untersucht. Auf den gesamten an der ETH Zürich durchgeführten Versuchen mit Granulat basiert eine von Heller et al. (2009) ausgearbeitete Bemessungstabelle, mit deren Hilfe in einfacher Weise erste Abschätzungen von Impulswellenparametern und dem Volumen, welches über eine Sperre schwappt, durchgeführt werden können. Dabei kann durch Definition der Einflussparameter des Rutsches 
(Eintauchgeschwindigkeit und -winkel, Abmessungen, Dichte und Porosität der Rutschmasse) und der Einflussparameter des Stauraums bzw. der Sperre (Distanz des Einstoßes zur Sperre, Wassertiefe, Freibord, Kronenbreite, Auflaufwinkel) die Auflaufhöhe sowie das Überschwappvolumen an einer Sperre recht schnell ermittelt werden (Gl. 1, Tab. 1). Nachteil dieses einfachen Bemessungsansatzes ist, dass nur einfache, generalisierte Geometrien des Stauraumes erfasst werden können. Eine Zusammenfassung des Excel-Tools, der zugrunde liegenden empirischen Ansätze sowie der bisher durchgeführten Modellversuche ist beispielsweise in Gabl et al. (2015) zu finden.

\subsection{Ansätze basierend auf 3D- numerischen Untersuchungen}

\subsubsection{Allgemein}

Wie auch schon im vorangegangen Kapitel erhebt der vorliegende Beitrag nicht den Anspruch auf vollständige Darstellung von Untersuchungen und Ansätzen zur Modellierung von Lawineneinstößen in Speicher. Es soll ein Überblick über die Entwicklung der letzten Jahre bei den Ansätzen zur numerischen Modellierung am Arbeitsbereich Wasserbau der Universität Innsbruck gegeben werden. Im Vordergrund steht dabei die kommerzielle 3D-numerische Berechnungssoftware FLOW-3D, welche ein breites Anwendungsfeld hat, wobei die Stärken besonders in der Modellierung von Freispiegelabflüssen liegen (Faber et al. 2012; Gabl et al. 2014b, 2015; Gems et al. 2014, 2016; Seibl et al. 2014; Krzyzagorski et al. 2016; Waldy et al. 2015). Die Software ist auf die Berechnung von maximal zwei Fluiden beschränkt, wodurch eine Berücksichtigung eines zusätzlichen Fluids in der Lawine neben Luft und Wasser eine entsprechende Adaptierung der $\mathrm{Nu}$ merik notwendig macht. Diese werden in weiterer Folge vorgestellt und diskutiert. Die Stärke der Simulation mit FLOW-3D liegt in der Berechnung einer freien Wasseroberfläche, welche nahezu ohne eine bei anderen Softwareprodukten notwendige Verfeinerung des Berechnungsnetzes auskommt. Zudem ermöglicht eine solche Berechnung, komplexe Gegebenheiten (Hochwasserentlastungsanlagen, Wellenbrecher, Felsstrukturen etc.) zu berücksichtigen. Für Details zur Software sei auf das
Nutzerhandbuch und auf entsprechende Beiträge, wie zum Beispiel Gabl et al. (2014a, 2015) und Gems et al. (2016), verwiesen.

\subsubsection{Validierung mit experimentellen Daten}

Ausgangspunkt der Beschäftigung mit der Thematik des Lawineneinstoßes in einen Speicher war ein physikalischer Modellversuch einer sich damals noch in Planung befindlichen Wehranlage, bei der unter anderem auch Untersuchungen $\mathrm{zu}$ dieser Fragestellung durchgeführt wurden. Die Versuchsergebnisse stellen Daten für eine numerische Vergleichsrechnung dar (Gabl et al. 2010, 2014b). Zur Beschleunigung der im Modellversuch nachgebildeten Lawine diente dabei eine geneigte Rinne, die knapp oberhalb des prognostizierten Einstoßpunktes der Lawine die Übergabe der Rutschmasse von der Rinne auf das nachgebildete Gelände ermöglichte. Die Eingangsdaten für die Lawine (Geschwindigkeit, Mächtigkeit, Dichte, Einstoßpunkt) stammen wie bei allen weiteren, nachfolgend beschriebenen Untersuchungen von gesonderten Untersuchungen des Sturzprozesses mit entsprechenden Modellen und Extrapolationsverfahren, welche vom Auftraggeber zur Verfügung gestellt wurden (Gabl et al. 2015). Mittels Wellenpegeln wurde im Zuge der Versuchsdurchführung die Mächtigkeit der Impulswelle im nachgebildeten Speicher punktförmig gemessen und zusätzlich das an der Wehranlage pro Stauklappe überschwappende Volumen erfasst. Zur Anwendung kamen beim Labormodell zwei unterschiedliche Modelllawinen, welche sich in ihrer Zusammensetzung und somit auch ihrer Dichte voneinander unterschieden. Eine Modellannahme bestand aus 900 Golfbällen (Dichte ca. $600 \mathrm{~kg} \mathrm{~m}^{-3}$ unter Berücksichtigung einer Porosität von $25 \%$ ), welche in der Rinne platziert wurden. Anschließend wurde eine Klappe schlagartig geöffnet, sodass die Golfbälle in den Speicher rutschen konnten. Um den Einfluss einer höheren Einstoßdichte zu untersuchen, wurde in einer weiteren Modellannahme ein kompakter Körper auf Rollen (Dichte ca. $700 \mathrm{~kg} \mathrm{~m}^{-3}$ ) verwendet. Im Hinblick auf die Möglichkeiten und Grenzen der numerischen Modellierung wurde im Weiteren versucht, die im Modellversuch beobachteten und gemessenen Prozesse mit der Software FLOW-3D zu simulieren. Dabei galt es, vergleichbare Ansätze für die numerische Simulation von solchen modellierten Einstößen zu finden. Für beide Konzepte erlaubt die Software FLOW-3D vergleichbare Modellansätze, die für diese Fragestellung genutzt werden können: (a) Partikelmodell und (b) bewegte Objekte (GMO - General Moving Objects).

Beim Partikelmodell, welches in Analogie zum Golfballmodell angewendet wurde, bestand und besteht bis zur aktuellsten Version die Einschränkung, dass eine volle Interaktion zwischen Wasser, Modellberandung, Gelände und Partikel zwar implementiert war, aber die gegenseitige Beeinflussung der Partikel nicht berücksichtigt wurde. Somit kommt es $\mathrm{zu}$ unrealistischen Bewegungsmustern und einer Fortbewegung der einzelnen Elemente, als wären sie alleine in der Rinne. Zur Abhilfe dieser Problematik wurde den Partikeln eine geringe Menge an Startwasser zugegeben, wodurch der Partikelstrom ausgerichtet werden konnte. Der Nachteil dieser Methode besteht darin, dass das zusätzliche Startwasser im Hinblick auf den definierten Einstoßimpuls der Lawine von den zugegebenen Partikeln abgezogen werden muss und somit ein zusätzlicher Freiheitsgrad gegeben ist.

Die selbstständige Beschleunigung eines bewegten Objektes (GMO) ist in FLOW-3D basierend auf der verwendeten FAVOR-Technik relativ einfach möglich. Dabei wird nicht wie bei anderen Softwareprodukten eine permanente Adaptierung des Berechnungsnetzes benötigt, sondern die Geometrie wird jeweils an das rechteckige Netz angepasst, welches für die gesamte Berechnung fixiert ist (Gabl et al. 2014a). Auch eine volle Interaktion zwischen dem bewegten Objekt und dem Fluid ist möglich. Für die Untersuchung des Lawineneinstoßes erwies sich der komplett freie Bewegungsmodus dabei als nicht zielführend. Zum einen benötigte die Beschleunigung in der Rinne vergleichsweise viel Rechenzeit und teilweise führten sehr kleinskalige, sich aufschaukelnde Kollisionsmuster zu einem nicht erwünschten Verhalten. Der Eintritt des vollständig freigegebenen Körpers (alle Freiheitsgrade offen und somit eine volle Interaktion möglich) in die Fluidoberfläche erwies sich aber als Hauptproblem, da in dem Fall die Gefahr besteht, dass die allererste Kollision mit dem Fluid nicht korrekt berechnet wird und das einstoßende Objekt vor dem eigentlichen Eintauch- 
vorgang unrealistisch stark abprallte. Um diese Schwäche des Modells zu umgehen und die Berechnungszeit zu reduzieren, wurde die Bewegung des Objektes vollständig vorgegeben und somit eine Reaktion des Wassers auf den ersten Impuls des Objektes konzentriert. Abgesehen von ungünstigen Reflexionen stellt diese primäre Welle in der Regel auch die maximale Impulswelle dar (Gabl et al. 2014a; Heller et al. 2009). Der Start der Berechnung erfolgt somit direkt vor dem Einstoß, wo die Endgeschwindigkeit und -richtung der Lawine direkt auf den Körper aufgebracht werden. Nach dem Wasserkontakt taucht die Lawine (bedingt durch das Abflachen der Speichersohle) in das Gelände ein und verschwindet somit aus dem Untersuchungsbereich. Ein Aufschwimmen, wie es beim Modellversuch ersichtlich war, kann damit nicht direkt berücksichtigt werden. Als Alternative ist auch ein Wechsel in den freien Bewegungsmodus des Lawinenkörpers nach dem direkten Eintritt denkbar.

Aus diesen Untersuchungen und dem Vergleich der numerischen Ergebnisse mit den Versuchsdaten konnte der Schluss gezogen werden, dass die jeweilige Modelllawine bzw. die aus dem Lawineneinstoß resultierende Impulswelle mit einer sehr hohen Genauigkeit nachgebildet werden konnte. Umgerechnet in den Naturmaßstab lag die Differenz in den gemessenen Wasserspiegellagen zwischen Modellversuch und Numerik im Bereich von Zentimetern. Der Unterschied in den Messergebnissen zwischen den beiden experimentellen Konzepten „Golfbälle“ und „Festkörper“ lag im Dezimeterbereich (wieder auf die Natur bezogen) und war damit vergleichsweise größer (Gabl et al. 2010, 2014b). Die Frage nach der Richtigkeit einer der beiden Varianten könnte in diesem Zusammenhang nur mit entsprechenden Naturdaten untersucht werden. Anhand solcher Messungen wäre es möglich, beide Modellkonzepte zu kalibrieren und entsprechend zu validieren.

\subsubsection{Festkörperlawine}

Mit dem Hintergrund einer sehr guten Übereinstimmung der 3D-numerischen Untersuchungen mit dem Modellversuch wurde die numerische Berechnungsvariante auch für weitere Fallbeispiele mit vergleichbaren Fragestellungen verwendet. Dabei wurde verstärkt das Festkörpermodell mit dem bewegten Körper (GMO) verwendet, da es im Gegensatz zum Partikelmodell sehr genau die Eingangsgrößen (Geschwindigkeit und Lawinenhöhe) ohne Kalibrierung einhält. Zudem entfällt beim Festkörpermodell die Notwendigkeit der Abbildung einer Beschleunigungsstrecke und einer, damit verbundenen, eventuell notwendigen Kalibrierung der Endgeschwindigkeit des Gemisches in einer Rinne. Vermieden wird dadurch auch der zusätzlich jeweils notwendige Nachweis, dass das frei gewählte Verhältnis der Mengen an „Beschleunigungsfluid“ und Partikeln und deren Eigenschaft keinen merklichen Einfluss auf das Ergebnis der Untersuchung hat. Die Festkörpervariante hat hingegen den Nachteil, dass die Lawine in unterschiedliche Bereiche unterteilt werden muss, die jeweils gleiche Bewegungsmuster aufweisen. Ein Überlappen unterschiedlicher Lawinenteile ist möglich, doch ist dieses Verfahren bei komplexen Topografien und einer aufgefächerten Lawine sehr aufwendig. Zusätzlich ist eine Massenerhaltung (in dem Fall ist dies durch die Erhaltung des verdrängten Volumens durch die Modelllawine $\mathrm{zu}$ ersetzen) durch das Eintauchen der Modelllawine in das Gelände und der fehlenden Möglichkeit das Objekt entsprechend anzupassen kaum realisierbar. Erschwert wird die numerische Berechnung zudem durch potenziell vermehrt auftretende abgeschlossene Wasserbereiche, die im Zuge des Bewegungsprozesses entstehen und zerdrückt werden. Dadurch kann die Qualität der numerischen Lösung leiden. Es kann sich zum Beispiel ein zusätzlicher Verlust von Fluidvolumen im Berechnungsgebiet ergeben. Die Anwendung von bewegten Objekten in Verbindung mit realitätsnahen Geländestrukturen verursacht einen deutlichen Mehraufwand durch die Erstellung von Teillawinen. Zudem muss der Nachweis der Unabhängigkeit der sich daraus ergebenden Lösung von dieser Wahl geführt werden. Dies ist ebenfalls mit einem relativ hohen Aufwand verbunden. Dadurch hat dieses Modellkonzept für zukünftige Projekte als prioritärer Ansatz zur Modellierung der Lawine an Attraktivität verloren.

\subsubsection{Wasserlawine}

Aus der Nachrechnung der im Modellversuch in den Speicher eingetragenen Golfbälle mit dem Partikel-Was-
ser-Gemisch wurde in einem weiteren Entwicklungsschritt das Konzept der reinen Wasserlawine entworfen, welches im Beitrag von Gabl et al. (2015) vorgestellt wurde. Dabei wird auf die Verwendung von Partikeln vollständig verzichtet und ausschließlich ein Fluidkörper im tatsächlichen Anbruchgebiet der Lawine platziert. Dies war durch die neue Softwareoption in FLOW-3D, Startwasser auch als stl-Datei zu definieren, möglich geworden. Als erster Schritt dieses Konzepts ist dabei darauf zu achten, dass trotz der höheren Dichte im Vergleich zu Schnee bzw. zur Lawine die idente Masse vorgegeben wird. Dadurch reduziert sich die Höhe des Startwassers entsprechend der Dichterelation. In einem ersten Durchlauf wird die Rechnung aus diesem vorgegebenen Zustand heraus gestartet und die Beschleunigung des Wassers bis zum Eintritt in den Speicher berechnet. Knapp oberhalb des Wasserspiegels des Speichers wird eine Kontrollebene definiert, wo die Modelllawine mit der als Eingangswert dienenden Lawinensimulation verglichen wird. Diese grundlegende Voruntersuchung zur Bestimmung der Lawinenparameter sollte bei einem möglichst geringen Wasserstand bzw. mit einem leeren Speicher durchgeführt werden. Damit wird verhindert, dass der Wasserspiegel im Speicher als Gelände interpretiert wird und es somit zu einem Aufstaueffekt kommt, welcher die Lawinenparameter beeinflusst. Die Interaktion mit dem Wasser wird in der weiteren Modellierung mit FLOW-3D ergänzt. Bei diesem ersten Durchlauf wird die Modelllawine im Vergleich zu den zu erzielenden Werten der Lawine eine deutlich $\mathrm{zu}$ hohe Geschwindigkeit aufweisen. Um dies zu korrigieren, wird ein ausgewählter Zeitpunkt nach dem ersten Berechnungsstart herangezogen. Die vordefinierte Wasserlawine hat sich dabei dem Gelände schon angepasst und wird in dieser Form wieder als neues Startwasser in eine erneute Berechnung importiert. Somit wird die Geometrieanpassung übernommen, aber die gesamte kinetische Energie entfernt. Aus diesem Zustand heraus wird wieder bis zum Einstoß gerechnet und erneut verglichen. Je nach dem Ergebnis wird ein früherer oder späterer Zeitpunkt herangezogen und damit die Wasserlawine kalibriert. Abhängig von der Lawine und dem Gelände kann meist schon nach einigen wenigen Iterationen eine gute Übereinstimmung des ersten Impul- 


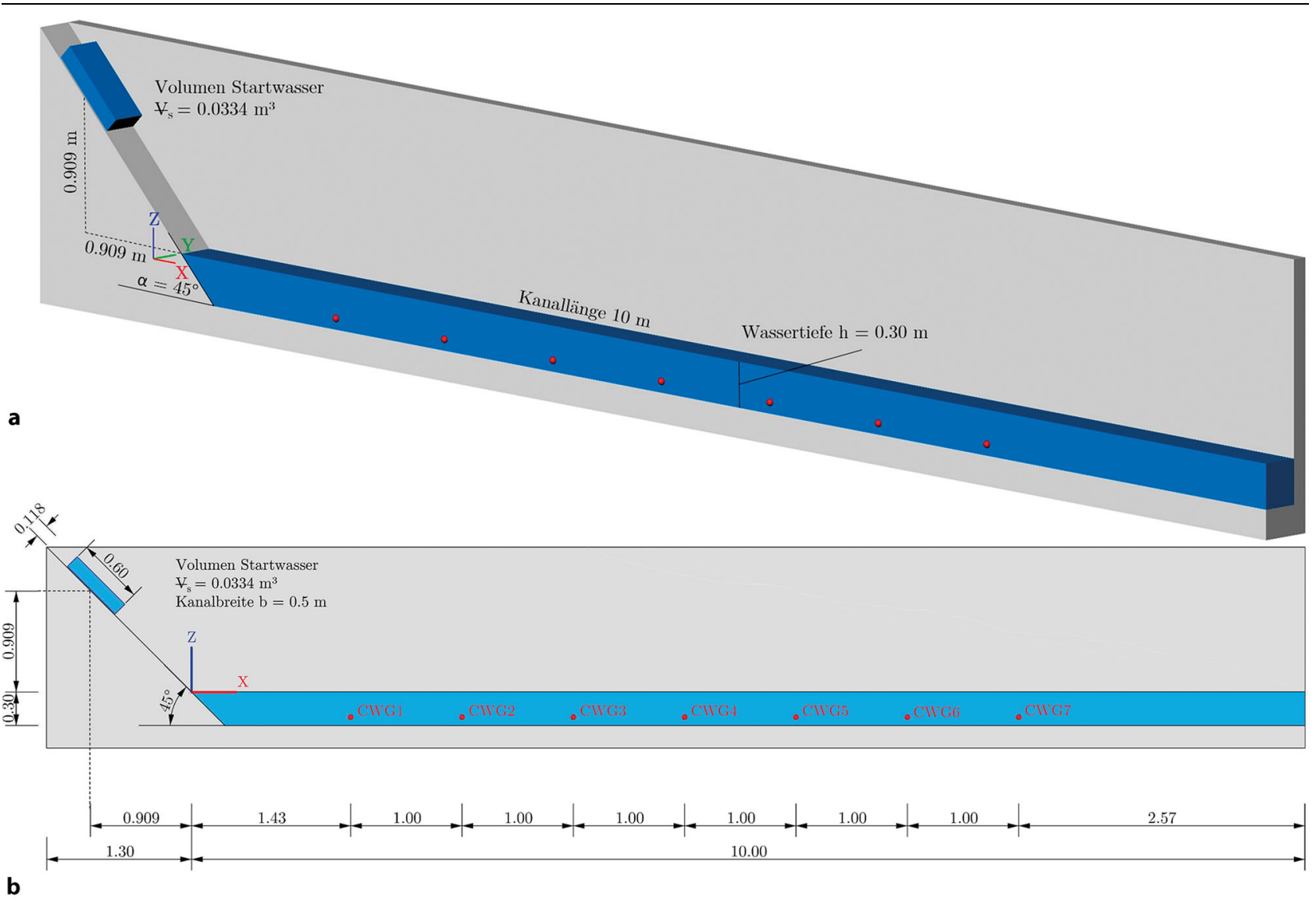

Abb. 1 Modellaufbau in Anlehnung an die Untersuchung von Heller (2009) - a Ansicht und b Aufriss

ses und auch dessen weitere zeitlichen Entwicklung festgestellt werden. Dies ist natürlich vom gegebenen Gelände abhängig und somit für jedes Projekt individuell zu überprüfen. Um eine Abhängigkeit der Berechnungsergebnisse von der anfänglich gewählten Startwasserverteilung im Anbruchgebiet der Lawine ausschließen zu können, sollte diese verändert werden und der Kalibrierungsprozess nochmals durchlaufen werden. Dadurch kann eine Modelllawine erzeugt werden, welche möglichst unabhängig von den gewählten Eingangsparametern ist und als Randbedingung für unterschiedlichste Untersuchungen der damit erzeugten Impulswelle im Speicher dient.

Um die Anwendbarkeit dieses Konzeptes nachzuweisen, wurden im Beitrag von Gabl et al. (2015) vereinfachte Rahmenbedingungen untersucht und die Ergebnisse mit der Ermittlung nach Heller et al. (2009) verglichen. Als Eingangswerte für die Untersuchung wurden dabei die Werte aus der 3D-Numerik verwendet. Somit wurden die vorangegangen numerischen Berechnungen mit den Formeln nachgerechnet. Im
Fokus der Untersuchung liegt dabei der Vergleich des jeweils überschwappenden Volumens bei der vereinfacht dargestellten Staumauer. Dies ist für die Beurteilung der Gefährdung des Absperrbauwerks und der Unterlieger entscheidend. Ergänzt wird die Untersuchung durch eine Parametervariation (vorhandenes Freibord, Wassertiefe im Speicher, Mauerhöhe und der Breite des Speichers in Relation zur einstoßenden Lawine). Als Schlussfolgerung aus diesem Vergleich an der vereinfachten Geometrie konnte eine sehr gute Übereinstimmung der 3D-numerischen Berechnung mit den Formeln nach Heller et al. (2009) festgestellt werden.

Basierend auf diesem Ansatz kann somit ein Lawineneinstoß als Randbedingung für eine Impulswellenausbreitung und Einwirkungsuntersuchung an einem Absperrbauwerk simuliert werden. Die Wasserlawine weist dabei bedingt durch die Kalibrierung einen im Vergleich zur nachzubildenden Lawine nahezu identischen Impuls auf. Der Massenerhalt im System ist ebenfalls gegeben, wobei Effekte wie allmähli- ches Abschmelzen oder Aufschwimmen nicht berücksichtigt werden können, da die Lawine schon als Wasser ankommt. Darüber hinaus passt sich die Modelllawine sehr gut dem Gelände an und es konnten vergleichbare Ergebnisse mit der ursprünglichen Lawinensimulation gefunden werden. Der wesentliche Unterschied ergibt sich aus den Werten für die Rutschmächtigkeit $s$ der Lawine an sich, welche beim numerischen Modellansatz (mit der Annahme von Wasser als Medium für den Lawinenprozess und der Massenerhaltung) deutlich geringer ausfällt. Bei der Anwendung des Konzepts der Wasserlawine wird deshalb empfohlen, die Formeln und Berechnungstabellen nach Heller et al. (2009) zum Vergleich heranzuziehen (Gabl et al. 2015). Die grundlegende Formel zur Bestimmung des Impulsproduktionsparameters $P$, aus dem sich alle weiteren Parameter (Tab. 1) bis hin zum Überschwappvolumen ermitteln lassen, ist wie folgt definiert (Heller et al. 2009): 


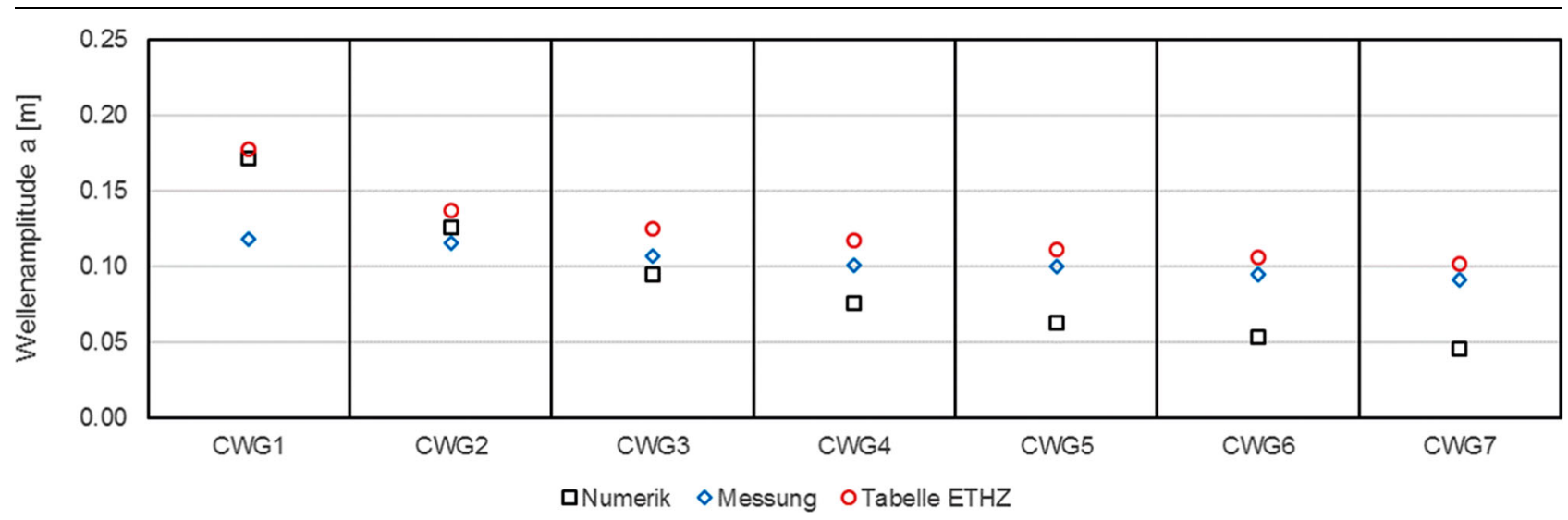

Abb. 2 Vergleich der Wellenamplitudean den sieben Messpunkten CWG1 bis CWG7-Numerik (FLOW-3D), Messung (Heller 2009), Berechnungstabelle der ETHZ (Heller et al. 2009)

a $\quad-0.3$

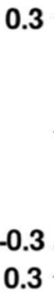

b

0.3

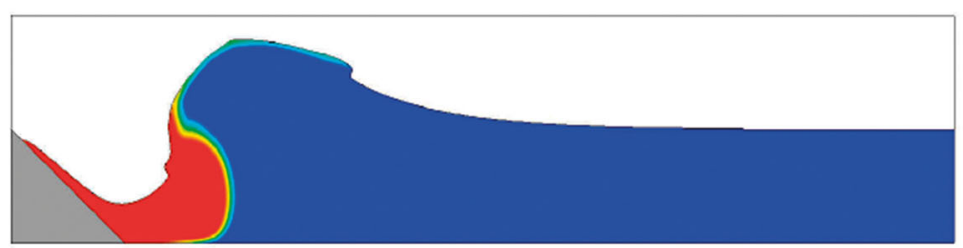

0.3

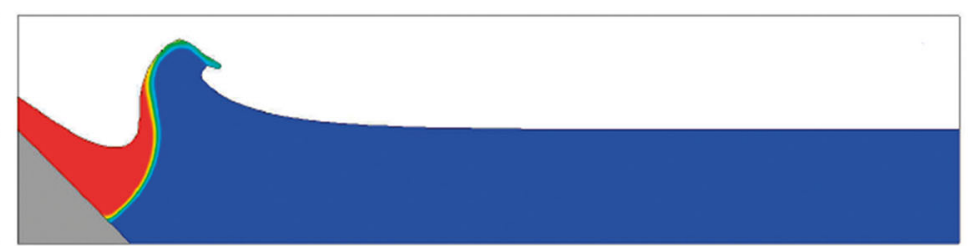

西

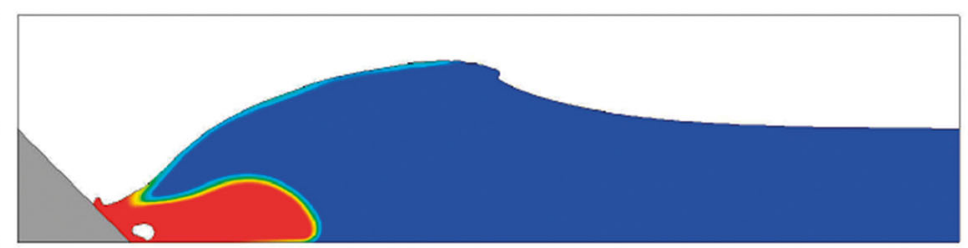

c $\quad-0.3$

0.3

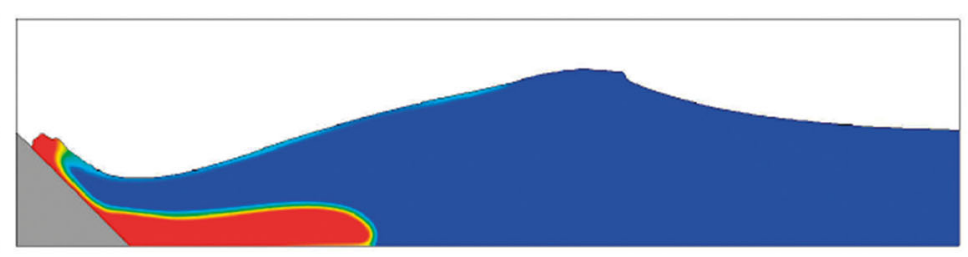

d -0.3

0.3

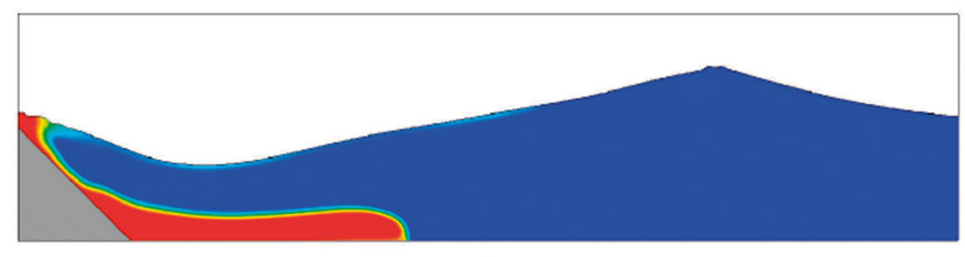

e

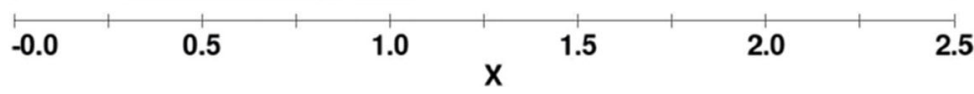

Abb. 3 Exemplarisches Ergebnis der Numerik mit FLOW-3D - Einstoß zu den Zeitpunkten (a) 0,2 s bis (b) 1 s in 0,2-Sekunden-Schritten - einstoßendes Wasser ist rot eingefärbt - Einheiten der Achsen in [m]

$$
\begin{aligned}
P= & \frac{v_{s}}{\sqrt{g h}} \cdot\left(\frac{s}{h}\right)^{1 / 2} \cdot\left(\frac{\rho_{s} V_{s}}{\rho_{W} b h^{2}}\right)^{1 / 4} . \\
& {\left[\cos \left(\frac{6}{7} \alpha\right)\right]^{1 / 2} }
\end{aligned}
$$

Im Gegensatz zur Rutschmächtigkeit $s$ gleicht sich der zweite Teil in Gl. 1 durch die Multiplikation der Rutschdichte $\rho_{\mathrm{s}}$ und des Rutschvolumens $\mathrm{V}_{\mathrm{s}}$ bei der Anpassung der Modelllawine aus. Aufbauend auf den im vorliegenden Beitrag angeführten numerischen Modellierungsansätzen wird in der Arbeit von Pfeifer (2016) die Möglichkeit einer Dichteanpassung des Startwassers im Anbruchgebiet untersucht. Dazu muss von der gewöhnlich getroffenen Annahme der Inkompressibilität des Wassers abgegangen werden und die Dichte als zusätzlicher Parameter in der Numerik freigegeben werden. In FLOW-3D ist dies mit der Aktivierung der „Density evaluation“ (Physics) möglich. Die Ergebnisse der ersten Untersuchungen mit dieser zusätzlichen Adaptierung sind in der Arbeit von Pfeifer (2016) im Detail nachzulesen und im nachfolgenden Kapitel zusammengefasst.

\section{Numerische Studie zur Möglichkeit der Dichtevariation des Lawinenmediums}

\subsection{Konzept und Aufbau des numerischen Modells}

Die Zielsetzung der vorgestellten Untersuchung (Pfeifer 2016) ist es, die prinzipielle Möglichkeit der Definition einer variablen Startdichte zur Verbesserung der in Gabl et al. (2015) beschriebenen Wasserlawine zu unter- 


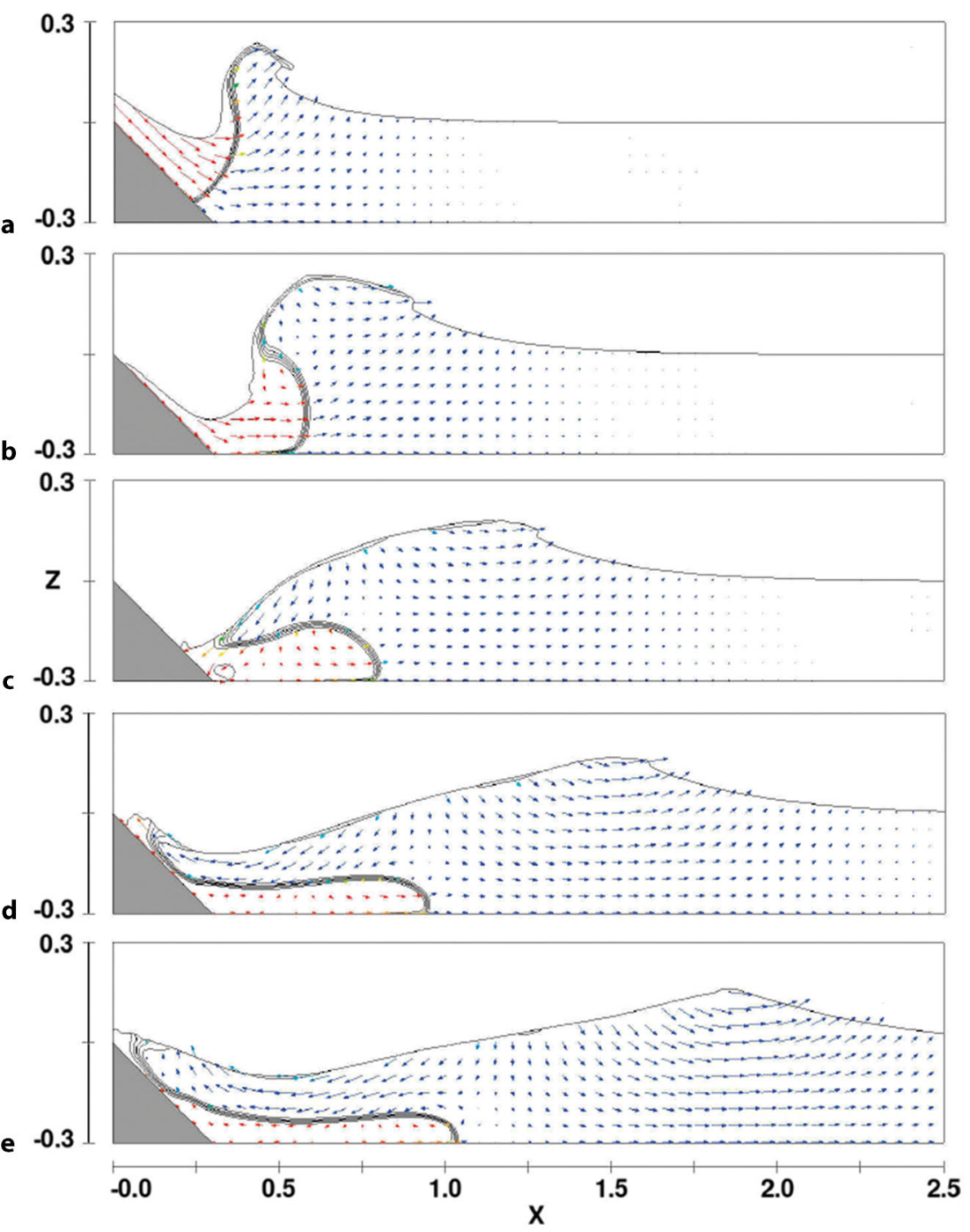

Abb. 4 Vektordarstellung zu Abb. 3 - Einheiten der Achsen in [m]

suchen und damit die Basis für eine weitere Modellentwicklung $\mathrm{zu}$ schaffen. Neben grundlegenden Voruntersuchungen zum Verhalten von Fluiden mit unterschiedlichen Dichten basierend auf der Anwendung von FLOW-3D wird dazu ein Validierungsexperiment herangezogen. Ausgangspunkt für die Untersuchung ist der von Dr. Valentin Heller zur Verfügung gestellte Benchmark-Test (Heller 2009), welcher auf der Internetseite von SPHERIC (SPH European Research Interest Community) veröffentlicht wurde. Der Datensatz soll bei (Weiter-)Entwicklungen im Bereich von SPH (Smoothed Particle Hydrodynamics) (Meister et al. 2014, 2015; Capone et al. 2010; Cascini et al. 2014; Heller et al. 2016) als Grundlage dienen und basiert auf einem typischen Rinnenversuch, wobei die Abmessungen in Abb. 1 dargestellt sind. Das einsto- zeichnet, wobei die Nummerierung in Einstoßrichtung erfolgt. Diese Punkte dienen als Referenzwerte für die Validierung der numerischen Berechnung. Die Lage der Kontrollpunkte und weitere Abmessungen sind in Abb. 1 dargestellt.

Die Untersuchung konzentriert sich auf die Entstehung und Ausbreitung der ersten Welle, welche direkt aus dem Einstoß erzeugt wird. Diese tritt an den untersuchten Punkten (CWG6 und CWG7) innerhalb der ersten acht Sekunden auf. Dadurch konnte auf die Modellierung des sich am Ende der Rinne befindenden Wellendämpfers verzichtet werden. In der numerischen Berechnung wurde an dieser Stelle eine stationäre Randbedingung in Form einer Wasserspiegellage definiert. Kontrolliert wurde dies durch eine Verlängerung des berechneten Beckens, was zu keinem unterschiedlichen Ergebnis führte. Alle anderen Randbedingungen sind als „Wand“ definiert bzw. durch die Geometrie beschränkt, wobei die Rauheiten des Modellversuches übernommen wurden. Als weitere Vereinfachung wird nur ein 2D-Schnitt des ursprünglich 0,5 m breiten Kanals berechnet. Dazu wird in der y-Richtung nur eine Zelle verwendet. Das Berechnungsnetz besteht nur aus einem einzigen Block, um eventuelle Interpolationsfehler beim Übergang von zwei Blöcken zu vermeiden. Im Zuge einer breiten Netzstudie wurde der Einfluss der Netzauflösung bei der räumlichen Diskretisierung untersucht und abschließend eine homogene Auflösung von $0,5 \mathrm{~cm}$ in allen Richtungen des Koordinatensystems gewählt. Eine weitere Verfeinerung der Zellen wäre wünschenswert, da noch keine vollständige Netzunabhängigkeit nachgewiesen werden konnte. Für den relativen Vergleich zwischen den zu untersuchenden Eingangsparameter kann der gewählte Wert aber als akzeptabel bezeichnet werden (Pfeifer 2016).

\subsection{Ergebnisse}

\subsubsection{Vergleich von numerischer Berechnung und Modellversuch}

Der Vergleich zwischen dem Benchmark-Test (Heller 2009) und den Ergebnissen aus der numerischen Berechnung ist in Abb. 2 dargestellt. Auf der Ordinatenachse ist dabei die Wellenamplitude an den sieben Messpunkten aufgetragen, welche sich aus der maximalen Abweichung zwischen der freien 


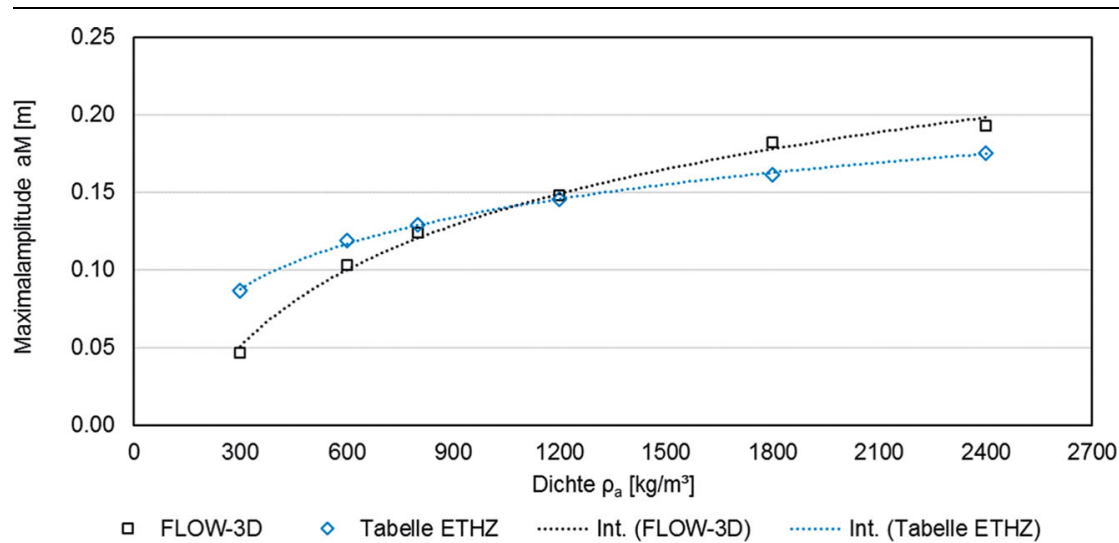

Abb. 5 Variation der Dichte der einstoßenden Modelllawine-der Ausgangswert liegt bei $1678 \mathrm{~kg} \mathrm{~m}^{-3}$

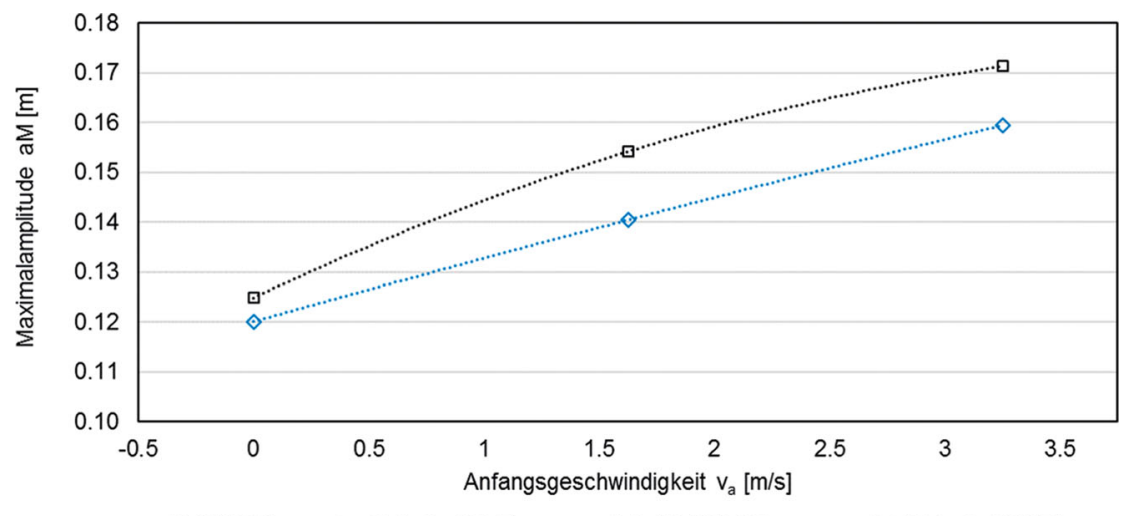

Abb. 6 Reduktion derStartgeschwindigkeitder einstoßenden Modelllawine-derAusgangswert liegt bei $3,25 \mathrm{~m} \mathrm{~s}^{-1}$

Wasseroberfläche bedingt durch die Welle in Relation zum Ruhewasserspiegel ergibt. Die Numerik überschätzt diesen Wert bis zum Pegel CWG3 und danach sind die numerisch ermittelten Abweichungen zum Ruhewasserspiegel zu gering. Schon im Zuge des Netztests konnte festgestellt werden, dass die Geometrie durch die zu berechnende schräge Ebene nicht ideal für die rechteckige Diskretisierung von FLOW3D ist (Gabl et al. 2014a). Mit jeder Verfeinerung des Netzes konnte eine bessere Auflösung der Geometrie erreicht werden, was wiederum mit einer Annäherung an die Messergebnisse verbunden war. Um die Abweichungen besser einschätzen zu können, wurde die auf den Eingangswerten des Modellversuches basierende Modelllawine vor dem Einstoß ausgewertet und dies als Grundlage für eine Abschätzung basierend auf Berechnungstabelle (Heller et al. 2009) durchgeführt. Dabei zeigt sich, dass diese Werte im Nahbereich des Einstoßes gut mit der Numerik
Verteilung erkennbar. Auch die Auswertung der Geschwindigkeiten zeigt eine deutlich stärkere Aufwärtsbewegung in der Numerik, als sie im Modellversuch aufgetreten ist. Dies lässt die Vermutung zu, dass neben der Dichte auch noch weitere Materialeigenschaften der Modelllawine angepasst werden müssten, um ein im Vergleich zum Granulat im Modellversuch möglichst ähnliches Verhalten $\mathrm{zu}$ erzielen. Zudem ist ein Einfluss der gewählten 2D-Betrachtung nicht ganz auszuschließen.

\subsubsection{Variation der Dichte und Startgeschwindigkeit}

Basierend auf den Erkenntnissen dieses Vergleiches von Messungen und Numerik werden zwei Parameter im numerischen Modell exemplarisch variiert. Dazu werden die Dichte der Modelllawine und zusätzlich auch die Startgeschwindigkeit zu Beginn der Berechnung untersucht. Die Dichte wird im Bereich von $300 \mathrm{~kg} \mathrm{~m}^{-3}$, was ein typischer Wert für Lawinen ist (Gabl et al. 2015), bis zu einem sehr dichten Gemisch mit $2400 \mathrm{~kg} \mathrm{~m}^{-3}$ untersucht. Die Startgeschwindigkeit wird im Vergleich zur Referenzgeschwindigkeit von ca. $3,25 \mathrm{~m} \mathrm{~s}^{-1}$ halbiert und in einer weiteren Rechnung auf $0 \mathrm{~m} \mathrm{~s}^{-1}$ gesetzt. Ziel ist es dabei, die Sensitivität der Ergebnisse in Abhängigkeit von den genannten Parametern zu erkennen. Als Vergleichsgröße wird in diesem Fall die Maximalamplitude $\mathrm{a}_{M}$ verwendet, welche die maximale Amplitude an allen sieben ausgewerteten Stellen darstellt. Dabei wird die gewählte Kombination der Eingangswerte jeweils zuerst mit FLOW-3D berechnet und ausgewertet. Die dabei ermittelten Kenngrößen der Modelllawine (Rutschmächtigkeit $s$ und Rutscheintauchgeschwindigkeit $v_{\mathrm{s}}$ ) sind bei Pfeifer (2016) im Detail aufgelistet und dienen wiederum als Input für die Abschätzung basierend auf der Berechnungstabelle nach Heller et al. (2009), welche als Vergleich jeweils ausgewertet wird.

Abb. 5 zeigt die Ergebnisse der Dichtevariation. Im Bereich geringer Dichten kommt es in der Numerik zu einer Unterschätzung der maximalen Wellenamplituden, Im Bereich hoher Dichten hingegen zu einer Überschätzung. Die besten Übereinstimmungen konnten mit einer Dichte von $1000 \mathrm{~kg} \mathrm{~m}^{-3}$ erzielt werden. In diesem Fall sind die Eigenschaften des einstoßenden Wassers ident mit jenen des ruhen- 
den Wasserkörpers. Die Reduktion der Startgeschwindigkeit hat im Vergleich dazu einen kleinen Einfluss auf die maximale Amplitude $a_{M}$ der Impulswelle im Speicher (Abb. 6).

\section{Zusammenfassung und Ausblick}

Neben der skalierten Nachbildung eine Lawineneinstoßes in einen Speicher im Labor bietet sich die 3D-Numerik als weiteres Werkzeug an. Besonders kann damit nach einer ersten Abschätzung mit den gängigen Berechnungsansätzen (Heller et al. 2009) eine Berücksichtigung von komplexeren Speicherformen oder auch Einbauten und Hochwasserentlastungen erfolgen. Dabei können die Stärken der 3D-numerischen Software FLOW-3D in Hinblick auf die Simulation von freien Wasseroberflächen und die einfache Integration von Geometrieanpassungen sehr gut genutzt werden. Der Beitrag zeigt die Entwicklung der Ansätze für die Modelllawinen in FLOW-3D, welche von Partikelgemischen über Festkörper bis hin zu reinen Wasserlawinen reicht. Die letzte Neuerung stellt dabei die Wasserlawine mit einer angepassten Startdichte dar. Dieser erweiterte Ansatz wurde hinsichtlich seiner prinzipiellen Umsetzbarkeit un- tersucht. Beim vorgestellten vereinfachten Experimentaufbau, welcher sich an einen Benchmark-Test anlehnt, stellten sich die Diskretisierung der schrägen Ebene und die Materialparameter der einstoßenden Modelllawine, die über die reine Dichte hinausgehen, als mögliche Quelle für die Abweichung zu den gemessen Werten heraus.

Als Ansatzpunkt für weitere Untersuchungen bietet sich die weitere Variation von Materialparametern der Modelllawine an und der Wechsel auf einen Versuchsaufbau, bei dem nicht eine schräge Ebene diskretisiert werden muss. Dies wäre auch durch eine Anpassung des lokalen Koordinatensystems lösbar (Gabl et al. 2014a), sodass das rechteckige Berechnungsnetz entlang der schrägen Ebene ausgerichtet ist. Damit könnte der Einfluss der Diskretisierung der schrägen Ebene untersucht werden.

Neben der Weiterentwicklung des Ansatzes mit der Software sind auch Simulationen mit SPH (Smoothed Particle Hydrodynamics)-Methode anzustreben. Die SPH-Methode kommt gegenwärtig bei vergleichbaren Aufgabenstellungen vermehrt zum Einsatz (Capone et al. 2010; Cascini et al. 2014; Heller et al. 2016), und deren Anwen- dung bringt deutliche Vorteile durch die Unabhängigkeit (das Fehlen) von Berechnungsnetzen mit sich. Das Hauptproblem in diesem Bereich wird aber auch weiterhin der Mangel an guten Kalibrierungsdaten aus Großversuchen sein.

Open access funding provided by University of Innsbruck and Medical University of Innsbruck.

\section{Interessenkonflikt}

R. Gabl, J. Seibl, M. Pfeifer, B. Gems und M. Aufleger geben an, dass kein Interessenkonflikt besteht.

Open Access Dieser Artikel wird unter der Creative Commons Namensnennung 4.0 International Lizenz (http:// creativecommons.org/licenses/by/4. $0 /$ deed.de) veröffentlicht, welche die Nutzung, Vervielfältigung, Bearbeitung, Verbreitung und Wiedergabe in jeglichem Medium und Format erlaubt, sofern Sie den/die ursprünglichen $\mathrm{Au}$ tor(en) und die Quelle ordnungsgemäß nennen, einen Link zur Creative Commons Lizenz beifügen und angeben, ob Änderungen vorgenommen wurden.
Bergmeister, K., Suda, J., Hübl, J., Rudolf-Miklau, F. (2009): Schutzbauwerke gegen Wildbachgefahren - Grundlagen, Entwurf und Bemessung, Beispiele. Berlin, Ernst \& Sohn.

Capone, T., Panizzo, A., Monaghan, J. J. (2010): SPH modelling of water waves generated by submarine landslides, J. Hydraul. Res., 48, 80-84, doi:10.1080/00221686.2010.9641248.

Cascini, L., Cuomo, S., Pastor, M., Sorbino, G., Piciullo, L. (2014): SPH run-out modelling of channelised landslides of the flow type, Geomorphology, 214, 502-513, doi:10.1016/j.geomorph. 2014.02.031.

De Cesare, G., Pfister, M., Daneshvari, M., Bieri, M. (2012): Herausforderungen des heutigen wasserbaulichen Versuchswesens mit drei Bei spielen (Challenges of current hydraulic modelling with three examples), WasserWirtschaft, 102/7-8, 71-75.

Czerny, H., Eistert, T. (2014): Bemessung und Überwachung von Beschneiungsspeichern (Assessment and monitoring of reservoirs for artificial snow production), Österreichische Wasser- und Abfallwirtschaft, 66/7-8, 271-278, doi: 10.1007/s00506-014-0164-0

Faber, R., Fuchs, M., Pucher, G. (2012): Numerische Simulation von Hochwässern: Von 1D zu 3D aus Anwendersicht im Ingenieurbereich (Numerical simulation of floods: From 1D to 3D from an applying engineer's view), Österreichische Wasser- und Abfallwirtschaft, 64/5, 307313, doi: 10.1007/s00506-012-0404-0.

Fuchs, H., Hager, W. (2012): Scale Effects of Impulse Wave Run-Up and Run-Over, J. Waterway,
Port, Coastal, Ocean Eng., 138(4), 303-311, doi: 10.1061/(ASCE)WW.1943-5460.0000138.

Fuchs, H., Heller, V., Hager, W. (2010): Impulse wave run-over: experimental benchmark study for numerical modelling, Experiments in Fluids, 49(5), 985-1004, doi:10.1007/s00348-010-0836$\mathrm{x}$.

Fuchs, H., Pfister, M., Boes, R., Perzlmaier, S. and Reindl, R. (2011): Impulswellen infolge Lawineneinstoß in den Speicher Kühtai (Impulse wineneinstoß in den Speicher Kühtai (Impulse
wave due to avalanche impact into Kühtai reservoir), WasserWirtschaft, 101/1-2, 54-60.

Gabl, R., Kapeller, G., Aufleger, M. (2010): Lawineneinstoß in einen Speichersee - Vergleich numerisches und physikalisches Modell (Avalanche Impact into a Reservoir - Comparison of Numerical and Physical Model), WasserWirtschaft, 100/5, 26-29, doi: 10.1007/BF03241644.

Gabl, R., Achleitner, S., Sendlhofer, A., Höck ner, T., Schmitter, M., Aufleger, M. (2013): Optimierter Einsatz und Kombination von 3-D-Numerik und physikalischer Modellierung (Combined use of 3D-numerics and scale model test), WasserWirtschaft, 103/5, 128-131, doi 10.1365/s35147-013-0550-8

Gabl, R., Gems, B., De Cesare, G., Aufleger, M. (2014a): Anregungen zur Qualitätssicherung in der 3-D-numerischen Modellierung mit FLOW3D (Contribution to Quality Standards for 3DNumerical Simulations with FLOW-3D), WasserWirtschaft, 104/3, 15-20, doi:10.1365/s35147014-0938-0.

Gabl, R., Gems, B., Plörer, M., Klar, R., Gschnitzer, T., Achleitner, S., Aufleger, M. (2014b): Numerical simulations in hydraulic engineer- ing, in: Computational Engineering, Dordrecht, Heidelberg, London, New York, Berlin, 195-224, doi:10.1007/978-3-319-05933-4_8.

Gabl, R., Seibl, J., Gems, B., Aufleger, M. (2015): 3-D numerical approach to simulate the overtopping volume caused by an impulse wave comparable to avalanche impact in a reservoir, Nat. Hazards Earth Syst. Sci., 15, 2617-2630, doi 10.5194/nhess-15-2617-2015.

Gems, B., Wörndl, M., Gabl, R., Weber, C., Aufleger, M. (2014): Experimental and numerical study on the design of a deposition basin outlet structure at a mountain debris cone, Nat. Hazards Earth Syst. Sci., 14, 175-187, doi:10.5194/ nhess-14-175-2014

Gems, B., Mazzorana, B., Hofer, T., Sturm, M. Gabl, R., Aufleger, M. (2016): 3D-hydrodynamic modelling of flood impacts on a building and in door flooding processes, Nat. Hazards Earth Syst. Sci., 16, 1351-1368, doi: 10.5194/nhess-16-13512016.

Heller, V (2009): Subaerial landslide generated impulse waves in a wave channel, http:// www.spheric-sph.org/tests/test-7 (Stand August 2016).

Heller, V., Spinneken, J. (2015): On the effect of the water body geometry on landslide-tsunamis: Physical insight from laboratory tests and $2 \mathrm{D}$ to $3 \mathrm{D}$ wave parameter transformation, Coastal Engineering, 104, 113-134, doi:10.1016/j.coastaleng.2015.06.006.

Heller, V., Hager, W. H., Minor, H.-E. (2008): Scale effects in subaerial landslide generated impulse waves, Exp. Fluids, 44, 691-703, doi:10.1007/s00348-007-0427-7. 
Heller, V., Hager, W. H., Minor, H.-E. (2009): Landslide generated impulse waves in reservoirs - Basics and computation, Mitteilungen 211, Versuchsanstalt für Wasserbau, Hydrologie und Glaziologie (VAW), ETH Zürich; Berechnungstabelle verfügbar unter http://www.vaw.ethz.ch/ das-institut/vaw-mitteilungen/2000-2009.html (Stand September 2016).

Heller, V., Bruggemann, M., Spinneken, J., Rogers, B. (2016): Composite modelling of subaerial landslide-tsunamis in different water body geometries and novel insight into slide and wave kinematics, Coastal Engineering, 109, 20-41, doi: 10.1016/j.coastaleng.2015.12.004

Kienholz, H., Krummenacher, B., Kipfer, A. Perret, S. (2004): Integrales Risiko Management in der Praxis - Bemerkungen zum Umgang mit Naturgefahren in der Schweiz (Aspects of integral risk management in practice - considerations with respect to mountain hazards in Switzerland), Österreichische Wasser und Abfallwirtschaft, 56/ 3-4, 43-50.

Krzyzagorski, S., Gabl, R., Seibl, J., Aufleger, M. (2016): Implementierung eines schräg angeströmten Rechens in die 3D-numerische Berechnung mit FLOW-3D (Implementation of an angled trash rack in the 3D-numerica simulation with FLOW-3D), Österreichische Wasser- und Abfallwirtschaft, 68/3, 146-153, doi 10.1007/s00506-016-0299-2.
Meister, M., Burger, G., Rauch, W. (2014): On the Reynolds number sensitivity of smoothed particle hydrodynamics, J. Hydraul. Res., 52, 824-835, doi:10.1080/00221686.2014.932855.

Meister, M., Fleischhacker, N., Rauch, W. (2015): Smoothed Particle Hydrodynamics - partikelbasierte Strömungsberechnung zur Anwendung in der Siedlungswasserwirtschaft (Smoothed particle hydrodynamics: particle-based fluid dynamics calculations for use in sanitary engineering), Österreichische Wasser- und Abfallwirtschaft, $67 / 3,148-152$, doi: 10.1007/s00506015-0217-z.

Möderl, M., De Toffol, S., Vanham, D., Fleischhacker, E., Rauch W. (2008): Abschätzung des Risikos von Naturgefahren für Wasserversorgungssysteme auf Basis der Systemvulnerabilität (Assessing the risk of natural hazards to watersupply systems on the basis of system vulnerability), Österreichische Wasser- und Abfallwirtschaft, 60/9-10, 149-155, doi: 10.1007/s00506008-0018-8.

Müller, C., Frank, P-J., Hager, W. (2016): Dyke overtopping: effects of shape and headwater elevation, J. Hydraul. Res, 54(4), 410-422, doi: 10.1080/00221686.2016.1170072.

Pfeifer, M. (2016): Untersuchung des Einflusses der Dichte bei einem Lawineneinstoß in einen Speicher. Masterarbeit am Arbeitsbereich Wasserbau der Universität Innsbruck, Begutachter Prof. M. Aufleger.
Ritter, A. (1892): Die Fortpflanzung der Wasserwellen, Zeitschrift des Vereins deutscher Ingenieure 36, 947-954.

Sauermoser, C., Lowatschek, G., Feda, T., Rudolf-Miklau, F (2016): Die Bauwerksdatenbank der Wildbach- und Lawinenverbauung. Nutzen für Gemeinden und Wassergenossenschaften (The Austrian service for torrent and avalanche control's structures database: benefits for municipalities and water associations), Österreichische Wasser- und Abfallwirtschaft, 68/1, 4653, doi:10.1007/s00506-015-0285-0.

Seibl, J., Gabl, R., Gems, B., Aufleger, M. (2014): 3-D-numerische Berechnung der Ausflusskurve infolge Staumauerversagen (3-D-numerical Investigation of the Outflow-Hydrograph for Dam Failure), WasserWirtschaft 104/11, 28-33, doi: 10.1365/s35147-014-1197-9.

Waldy, M., Gabl, R., Seibl, J., Aufleger, M. (2015): Alternative Methoden für die Implementierung von Rechenverlusten in die 3D-numerische Berechnung mit FLOW-3D (Alternative methods for the implementation of trash rack losses in the 3D-numerical calculation with FLOW-3D), Österreichische Wasser- und Abfallwirtschaft, 67/1, 64-69, doi: 10.1007/s00506-014-0205-8 Zweifel, A. (2004): Impulswellen: Effekte der Rutschdichte und der Wassertiefe, Mitteilungen 186, Versuchsanstalt für Wasserbau, Hydrologie und Glaziologie (VAW), ETH Zürich. 